\title{
Homotypic and heterotypic protein associations control Rad51 function in double-strand break repair
}

\author{
John W. Donovan, G. Todd Milne, and David T. Weaver ${ }^{1}$ \\ Division of Tumor Immunology, Dana-Farber Cancer Institute, Department of Microbiology and Molecular Genetics, \\ Harvard Medical School, Boston, Massachusetts 02115 USA
}

\begin{abstract}
Rad51 is essential for efficient repair of DNA double-strand breaks (DSBs) and recombination in Saccharomyces cerevisiae. Here, we examine Rad51 protein-protein interactions and their biological significance. GAL4 two-hybrid fusion analysis demonstrated that the amino-terminal region of Rad51 mediates both a strong Rad51:Rad51 self-association and a Rad51:Rad52 interaction. Several Rad51 variants were characterized that imparted DSB repair defects; these defects appear to result from Rad51 protein-protein interactions. First, a rad51 allele bearing a missense mutation in the consensus ATP-binding sequence disrupted DSB repair in wild-type yeast. The effect of this allele was dependent on the presence of wild-type Rad51 because MMS sensitivity of rad51 $\triangle$ strains were not increased by its expression. Second, we identified a highly conserved RAD51 homolog from Kluyveromyces lactis (KIRAD51) that only partially complemented rad51 $\triangle$ strains and impaired DSB repair in wild-type $S$. cerevisiae. Third, fusions of Gal4 domains to Rad51 disrupted DSB repair in a manner that required the presence of either Rad51 or Rad52. Because $K$. lactis $R A D 51$ and $R A D 52$ did not complement a $S$. cerevisiae rad51 $\Delta$ rad52 $\Delta$ strain, Rad51-Rad52 functions appear to be mediated through additional components. Thus, multiple types of Rad51 protein interactions, including self-association, appear to be important for DSB repair.
\end{abstract}

[Key Words: RAD51; RAD52; double-strand break repair; self-association; negative dominance]

Received August 5, 1994; revised version accepted September 20, 1994.

The repair of damaged DNA is critical for the faithful transmission of genetic information and the maintenance of genomic stability. In Saccharomyces cerevisiae, nearly 100 loci have been identified that are required for this process. On the basis of double mutant analysis, these loci can be divided into three epistasis groups that control nucleotide excision repair $(R A D 3$ epistasis group), error-prone/post-replication repair (RAD6 epistasis group), and recombinational repair (RAD52 epistasis group; for review, see Game 1983; Friedberg 1991; Petes et al. 1991). The third class of genes, which include RAD50-57, MRE2, MRE11, and XRS2, is primarily responsible for the repair of DNA double-strand breaks (DSBs). RAD52 epistasis group mutants exhibit sensitivity to agents that generate DSBs and have pleiotropic defects in mitotic and meiotic recombination.

Rad51 from yeast and other eukaryotes has considerable homology to bacterial RecA (Aboussekhra et al. 1992; Basile et al. 1992; Shinohara et al. 1992; Bezzubova et al. 1993; Morita et al. 1993; Muris et al. 1993; Shinohara et al. 1993; Yoshimura et al. 1993; Jang et al. 1994). Computer modeling indicates that there is significant structural similarity between these proteins as well, sug-

${ }^{1}$ Corresponding author. gesting a conserved function in DSB repair (Story et al. 1993). In Escherichia coli, RecA plays a central role in recombinational DNA repair (for review, see Roca and Cox 1990; Kowalczykowski 1991; Radding 1991). RecA promotes the pairing and exchange of DNA strands between homologous sequences by forming a helical protein filament that is a product of both RecA-DNA interactions and RecA self-association (Stasiak et al. 1988). Rad51 also forms a helical filament on double-stranded DNA in vitro (Ogawa et al. 1993). The rad51-10 allele, which is semidominant in a heterozygous diploid, suggests that Rad51, like RecA, self-associates (Aboussekhra et al. 1992). Additionally, Rad51, like RecA, binds DNA in the presence of ATP and is a DNA-dependent ATPase (Shinohara et al. 1992). A compelling hypothesis is that $\operatorname{Rad} 51$ is also biologically active as a nucleoprotein filament.

The epistatic interactions of $R A D 52$ group genes reflect their function in a common repair pathway. Moreover, there appears to be physical association of at least a subset of the gene products. Genetic and physical evidence for the interaction between Rad52 and Rad51 has been reported (Shinohara et al. 1992; Milne and Weaver 1993). Additional evidence for a multiprotein repair complex comes from the negative dominance exerted by 
mutant alleles of $R A D 52$. Some rad52 dominant-negative alleles act via nonproductive interaction with Rad51, whereas others can act independently of Rad51, perhaps by associating with other repair proteins (Milne and Weaver 1993). Thus, DSB repair in S. cerevisiae may involve a multiprotein complex.

Protein complexes control a variety of biological processes. Efficient DNA replication in phage T4 and E. coli require the assembly of holoenzyme complexes that includes both DNA polymerases as well as accessory proteins (McHenry 1991; Munn and Alberts 1991; Kornberg and Baker 1992; Young et al. 1992). In yeast, a multiprotein origin recognition complex (ORC) has been described that may regulate initation of DNA replication (Bell and Stillman 1992; Li and Alberts 1992). Large RNA-protein complexes (splicesomes) control RNA splicing in eukaryotes (Lamond 1993). Additionally, other DNA repair processes, including excision repair (Sancar and Sancar 1988; Bardwell et al. 1993) and methyl-directed mismatch repair (Modrich 1991), utilize multiprotein complexes. As with DNA replication, RNA splicing, and other types of DNA repair, DSB repair and recombination are processes that likely involve higher order protein complexes.

Here, we show that both Rad51 homotypic and heterotypic associations appear to govern Rad51 function in DSB repair in $S$. cerevisiae. An ATP-binding mutant, a Kluyveromyces lactis RAD51 homolog, and GAL4$R A D 51$ fusions all dominantly interfere with DSB repair when expressed in wild-type cells. This dominant interference requires either Rad51 or Rad52 for maximal effect. Both Rad51:Rad51 self-association and Rad51:Rad52 heterotypic interaction epitopes are localized to an amino-terminal region of Rad51 defined by GAL4 two-hybrid protein fusions. Our results strongly argue that Rad51 functions require self-association and interaction with other proteins for DSB repair and support a model where Rad51 functions in a multiprotein complex.

\section{Results}

\section{A dominant-negative allele of RAD51}

Physical evidence of RecA-like Rad51-DNA filaments (Ogawa et al. 1993) indicates that protein-protein interactions should be important for Rad51 function. These
Rad51 interactions could be revealed by the action of mutant alleles. A chromosomal mutation (K-A191) in the ATP-binding site of Rad51 results in a DNA repair and recombination deficiency in mutant yeast (Shinohara et al. 1992). We reasoned that rad51K-A191, although biologically inactive, should retain the capacity for protein-protein interactions and thus may dominantly interfere with DSB repair when expressed in wildtype cells.

To examine the ability of rad51K-A191 to dominantly interfere with DNA repair, we measured the DSB repair phenotypes of wild-type strains expressing this mutant allele. $R A D 51$ and rad51K-A191 alleles were transcribed from the $A D H$ promoter on a $2 \mu$ plasmid transformed into a wild-type (DWY83; see Table 1) and a rad51s strain (DWY120). Transformants were plated on solid media with and without methyl methane sulfonate (MMS) to measure DSB repair, and surviving colonies were scored over 5 days (see Materials and methods). Transformation of rad51K-A191 failed to complement the MMS sensitivity of the rad51 $\Delta$ strain. These transformants were indistinguishable from DWY120 transformed with vector alone (Fig. 1A). In contrast, transformation with wild-type $R A D 51$ conferred complete resistance to this concentration of MMS (Fig. 1A).

The DNA repair phenotype of DWY83 (wild type) transformed with $R A D 51$, rad51K-A191, or vector only was also measured on MMS selection plates. DWY83 transformants with vector control or wild-type RAD51 were equally MMS resistant, indicating that Rad51 overexpression does not influence DNA repair (Fig. 1B). However, transformation with rad51K-A191 significantly reduced the colony number on MMS plates over 5 days, indicating that repair has been disrupted. Several other haploid and diploid wild-type strains were similarly sensitive to rad51K-A191 expression (data not shown). Importantly, rad51K-A191 expression had no effect on the repair of UV damage in these cells, demonstrating a selectivity in inhibiting DSB repair (data not shown). The rad51K-A191 gene product, therefore, clearly has a strong dominant-negative effect on DSB repair when expressed in wild-type cells.

The amino-terminal region of Rad51 is important for self-association and interaction with Rad52

The simplest model that would describe the effect of

Table 1. Yeast strains used in this study

\begin{tabular}{|c|c|c|}
\hline Strain & Genotype & Source \\
\hline GGY1::171 & 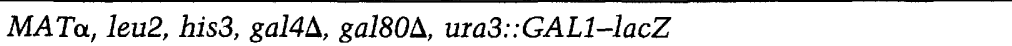 & Chien et al. (1991) \\
\hline DWY83 & $M A T \alpha, \arg 4-R V, \operatorname{leu} 2-3,112, \operatorname{cyh}^{R}$, ura3-52, $\operatorname{trp} 1-189$ & $\begin{array}{l}\text { R. Kolodner, Harvard Medical School, } \\
\text { Cambridge, MA }\end{array}$ \\
\hline DWY120 & MATa, ho::LYS2, lys2, leu2::hisG, ade2::LK, his4-XB, rad51 $::$ his $G$, ura3 & Milne and Weaver (1993) \\
\hline DWY127 & MATa, leu2-3,112, trp1-1, can1-100, ura3-1, ade2-1, his3-11,15 $\left[\mathrm{phi}^{+}\right]$ & $\begin{array}{l}\text { R. Rothstein, Columbia University; } \\
\text { NY }\end{array}$ \\
\hline DWY129 & MATa, rad51::LEU2, can1-100, his3-11,15, leu2-3,112, trp1-1, ura3-1, ade2-1 & $\begin{array}{l}\text { R. Rothstein, Columbia University, } \\
\text { NY }\end{array}$ \\
\hline DWY144 & rad51::LEU2, ura3, rad52A::ura3, Ieu2-3,112, his3-11,15 & this study \\
\hline
\end{tabular}




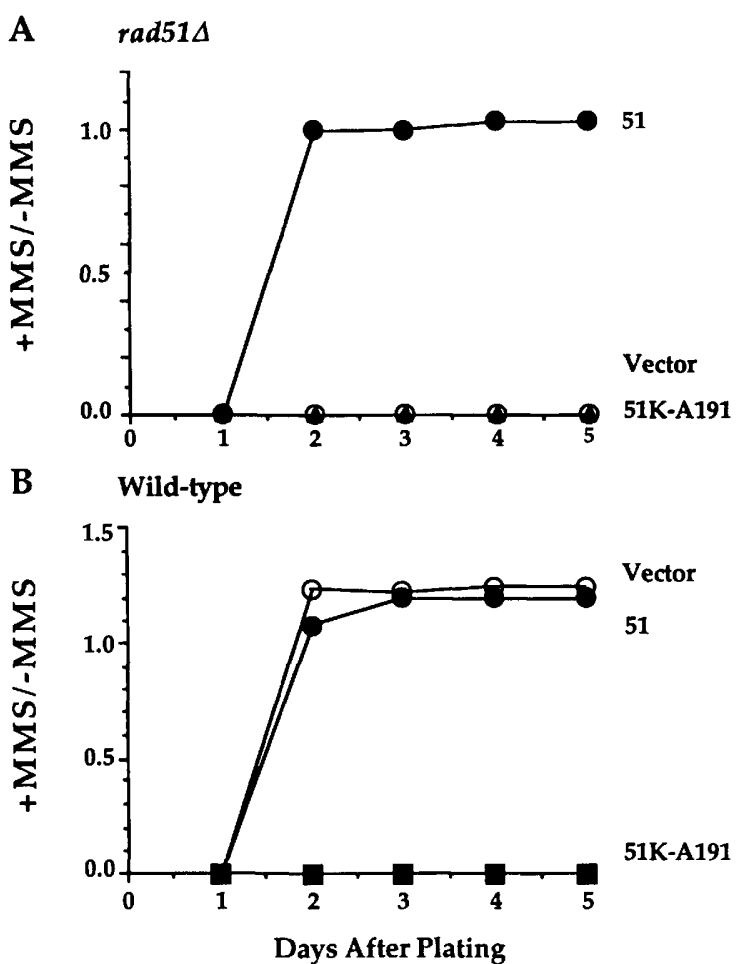

Figure 1. Rad51K-A191 dominantly interferes with DSB repair in wild-type $S$. cerevisiae. MMS sensitivity was determined by plating serial dilutions of transformants onto selective media with and without the appropriate concentration of MMS (see Materials and methods). The ratio of surviving colonies on MMS plates to colonies on plates without MMS over a period of 5 days is shown. $(A)$ Overexpression of rad51K-A191 does not complement the MMS sensitivity of a rad51 $\Delta$ strain. DWY120 (rad514) was transformed with pDB20 (Vector; O), $A D$. $H: R A D 51(51 ; 0)$, and $A D H: r a d 51 K-A(51 \mathrm{~K}-\mathrm{Al} 191 ; \Delta)$ and assayed on plates lacking uracil with $0.005 \%$ MMS. $(B)$ Overexpression of rad51K-A191 sensitizes wild-type cells to MMS. DWY83 (RAD51) was transformed with pDB20 (Vector; O), $A D$ $H: R A D 51(51 ; 0)$, and $A D H: r a d 51 K-A 191(51 \mathrm{~K}-\mathrm{Al} 191 ; \mathbf{0})$ and assayed on plates lacking uracil with $0.0025 \%$ MMS.

rad51K-A191 expression on MMS repair is that Rad51K-A191 is associating with endogenous proteins. To directly examine Rad51 protein-protein interactions in vivo, we used the GAL4 two-hybrid fusion system (Chien et al. 1991). For this assay, a gene is fused to both the DNA-binding and trans-activation domains of the yeast Gal4 protein and these fusions are coexpressed in a yeast strain containing a $l a c Z$ reporter gene downstream of a $G A L$ operator (GGY1::171). If the fusion protein(s) interact, the DNA-binding and trans-activation domains of Gal4 become tethered and can trans-activate the $1 a c Z$ reporter gene. Protein-protein interactions can be scored either by growth on plates containing X-gal or by measuring $\beta$-galactosidase activity in yeast extracts (see Materials and methods).

Plasmids were constructed in which the Gal4 DNAbinding domain (DB, amino acids 1-147) and the Gal4 transcriptional trans-activation domain (TA, amino ac- ids 768-881) were fused to the amino terminus of fulllength $\operatorname{Rad} 51$. GGY1::171 transformants coexpressing these two Rad51-Gal4 fusions (DB51 and TA51) were replica plated onto X-gal plates and colonies turned deep blue overnight. Extracts from these transformants were very active for $\beta$-galactosidase activity $(289$ Miller units of activity relative to 658 units for a GAL4 control) (Fig. 2A). Thus, the fusion proteins strongly interact in vivo. Neither Rad51-Gal4 fusion had an intrinsic trans-activation capacity, as the expression of either DB51 or TA5l with a vector control showed a background level of $\beta$-galactosidase activity $(<1.0$ Miller units). Also, no blue colonies or $\beta$-galactosidase activity above background were detected when TA51 was coexpressed with a fusion of the mammalian p53 gene to the DNA-binding domain of Gal4 (DBp53), indicating that fused Rad51 proteins do not interact promiscuously with other proteins (Fig. 2A).

We used the two-hybrid system to localize the region(s) necessary for Rad51 self-interaction. A series of truncated GAL4-RAD51 fusions were constructed and cotransformed into GGY1::171 with either of the fulllength $R A D 51$ fusion plasmids (DB51 or TA51) and monitored for $I a c Z$ expression on X-gal plates. The truncated Gal4 fusions 51A, 51C, 51D, 51E, and 51G, which retain the amino-terminal one-third of the Rad51 protein to varying degrees, each scored positive for self-association (Fig. 2B). Fusions 51A, 51C, 51D, 51E, and 51G overlap in a small region corresponding to the 33 amino acids encoded within the Bsu36I and NdeI sites as shown. To determine whether if this 33-amino-acid region was solely responsible for Rad51 self-association, we prepared the fusion protein $51 \mathrm{H}$ where the 33 amino acids were removed by an in-frame deletion (amino acids 78112). Fusion $51 \mathrm{H}$ gave blue colonies when coexpressed with a full-length Rad51 fusion. It appears that the interaction domain is either larger than amino acids 78112 and can be split, or removal of this domain reveals a second region of interaction. We conclude that the amino-terminal third of Rad51 is involved in self-association and that this association is probably not restricted to a single epitope.

Our observation that rad51K-A191 dominantly interferes with MMS repair in wild-type cells (Fig. 1B) suggests that inactive repair complexes may result from the interaction of mutant and wild-type Rad51. Thus, we examined the ability of Rad51K-A191 to associate with Rad51 in vivo. rad51K-A191 fusions to either GAL4 TA or DB (DB51K-A191 and TA51K-A191) were cotransformed into GGY1::171 with the corresponding wildtype or K-A191 mutant fusions. Both DB51K-A191 and TA51K-A191 strongly interacted with either mutant or wild-type fusion proteins at a level equivalent to the wild-type Rad51:Rad51 association (Fig. 2A; data not shown). Therefore, the K191 residue, and presumably ATP binding, are not necessary for Rad51 self-association in vivo.

We have shown previously that Rad51 and Rad52 can interact physically in vivo via the carboxy-terminal third of Rad52 and that this association is necessary for DNA repair (Milne and Weaver 1993). We used the truncated 


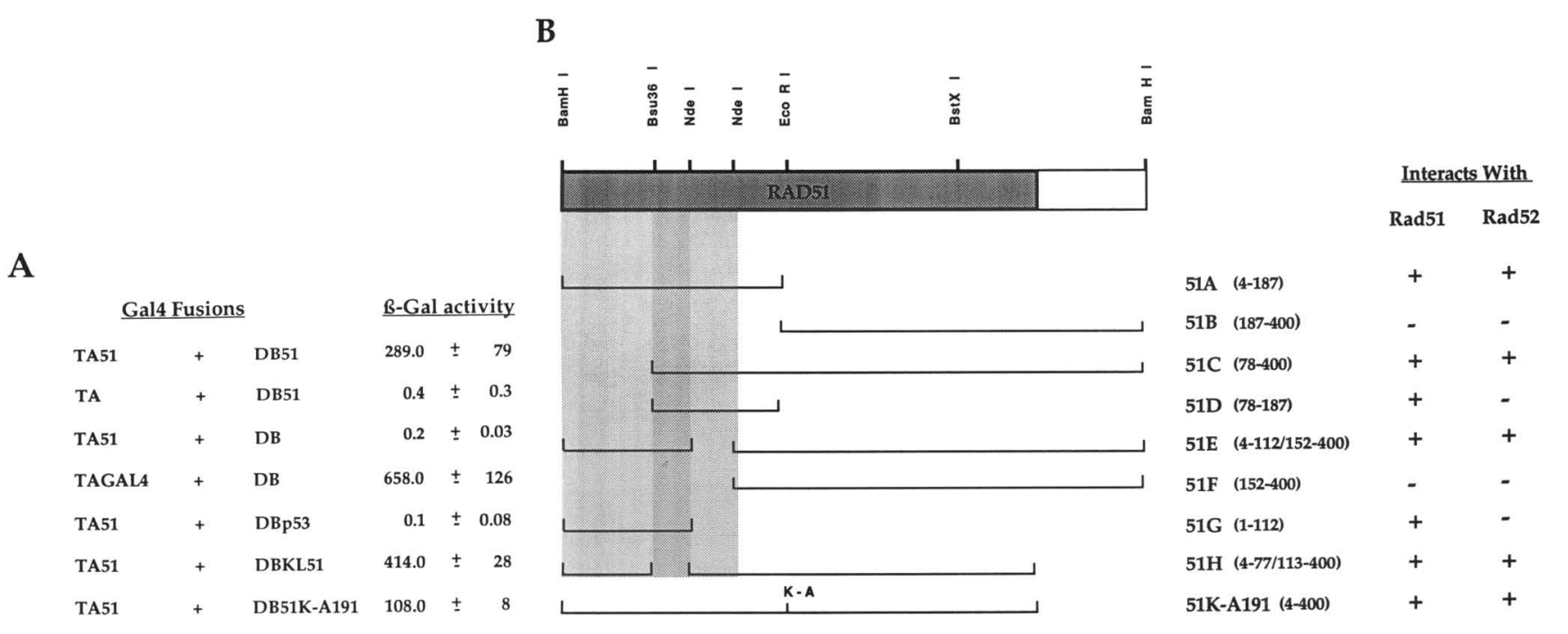

Figure 2. Rad51 self-associates via a domain in the amino terminus. GGY1::171 was cotransformed with plasmids expressing the indicated fusion proteins. $\beta$-Galactosidase activity (in Miller units) was quantitated as described in Materials and methods. (A) Rad51 physically interacts with Rad51, KlRad51, and Rad51K-A191 in vivo. (B) Rad51 self-association and interaction with Rad52 maps to the amino-terminal region. Fragments of RAD51 (amino acid numbers of fragments in parentheses) fused to the DNA-binding or trans-activation regions of GAL4 coexpressed with either TA51 or DB51, and TA-52 or DB-52 localize the protein-protein interaction to the amino terminus of Rad51. Transformants were scored + for interaction when blue colonies appeared on X-gal plates within 1 week of replica plating. The RAD51 gene (shaded) and 3' flanking sequences are shown boxed. Darkly shaded area indicates the region common to most truncated fusions. Lightly shaded area indicates region where additional sequences involved in protein-protein interactions are located. (K-A) The location of the substitution of lysine to alanine at amino acid 191.

Gal4-Rad51 fusions with Gal4-Rad52 fusions (DB-52 and TA-52) to map the region(s) of Rad51 that interact with Rad52. Blue colonies were observed when DB-52 or TA-52 were coexpressed with either $51 \mathrm{~A}, 51 \mathrm{C}, 51 \mathrm{E}, 51 \mathrm{H}$, and $51 \mathrm{~K}-\mathrm{A} 191$ but not with $51 \mathrm{~B}, 51 \mathrm{D}, 51 \mathrm{~F}$, or $51 \mathrm{G}$ (Fig. 2B). Thus, the pattern of Rad52:Rad51 interaction is very similar to that demonstrated for Rad51 self-association and suggests that the Rad52:Rad51 interacting region is near or coincident with that of Rad51 self-association.

\section{$\mathrm{K}$. lactis RAD51 causes a dominant-negative MMS repair phenotype in $\mathrm{S}$. cerevisiae}

Examining the function of RAD52 epistasis group genes from other budding yeasts in $S$. cerevisiae can reveal activities that are conserved versus those that are species specific in DSB repair. For example, the $K$. lactis RAD52 homolog complements $S$. cerevisiae rad52 $\Delta$ strains only weakly and dominantly interferes with DSB repair and recombination when overexpressed in wild-type cells (Milne and Weaver 1993). To identify a K. lactis homolog of RAD51, a genomic Southern blot with $K$. lactis DNA was probed with the $S$. cerevisiae RAD51 gene. We found a single cross-hybridizing band that was subsequently cloned from a size-fractionated $\mathrm{XbaI} K$. lactis genomic library (Materials and methods). The entire 1065-bp KIRAD51 open reading frame was located on a $4.5-\mathrm{kb}$ $X b a I-H i n d I I I$ subfragment and the DNA sequence determined. KIRad51 and ScRad51 are remarkably well con- served, showing $81 \%$ overall amino acid identity and $86 \%$ similarity (Fig. 3A). Most of the differences between KIRad51 and ScRad51 are in the amino-terminal 80 amino acids, where there is only $46 \%$ identity. The remainder of the protein, which corresponds to the region of Rad51 with the strongest homology to RecA, shows $>90 \%$ identity. KIRad51 is considerably more homologous to $S c \operatorname{Rad} 51$ and $\operatorname{Rad} 51$ proteins from $S$. pombe, mouse, chicken, and human, than to other $S$. cerevisiae RecA homologs [Rad55, Rad57, and Dmc1 (Fig. 3B; data not shown)]. Therefore, KlRad51 is a Rad51 homolog.

To examine the activity of KlRAD51 in S. cerevisiae, an ARS CEN plasmid with KIRAD51 expressed from the KIRAD51 promoter (pRSK151) was transformed into a rad51 $\Delta$ strain (DWY120) and tested for MMS repair activity. KIRAD51 only partially complemented DWY 120 compared with the wild-type $S$. cerevisiae RAD51 gene (Fig. 4A). The lack of full complementation could be attributable to poor expression of KIRad51 in S. cerevisiae; however, KlRAD51 expressed from the $S$. cerevisiae $A D H$ promoter on a multicopy plasmid complemented only slightly better (data not shown). Thus, the poor complementation provided by KIRAD51 is more likely a function of its weak intrinsic activity in $S$. cerevisiae, rather that poor expression. KIRad51 may be only partially active because of its inability to interact productively with $S$. cerevisiae repair proteins.

To determine whether KIRAD51 expression has a dominant-negative effect on DNA repair in S. cerevisiae, we transformed the wild-type strain DWY83 with KIRAD51, RAD51, and vector alone and measured MMS 
A

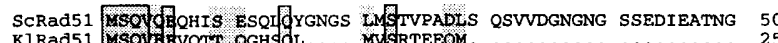

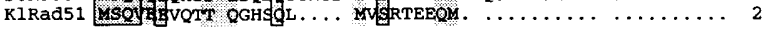

SCRad51 SGDGGGIPEQ AZAQG前EA A.

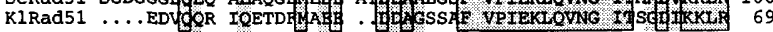

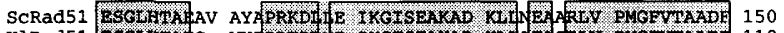

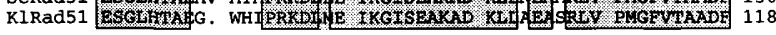

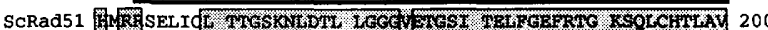

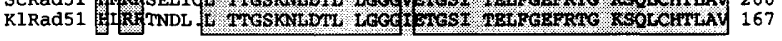

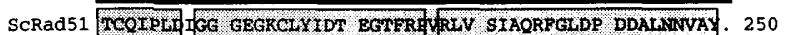

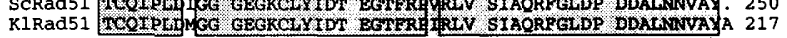

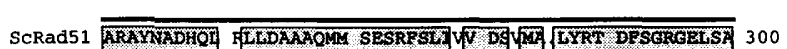

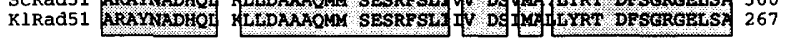

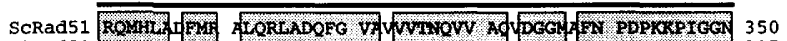

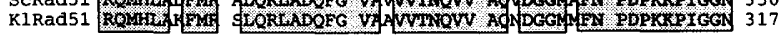

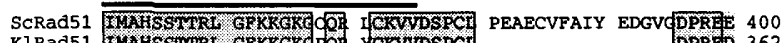

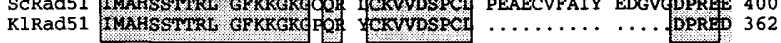

ScRad51 A 402

K1Rad51 . . 364

B

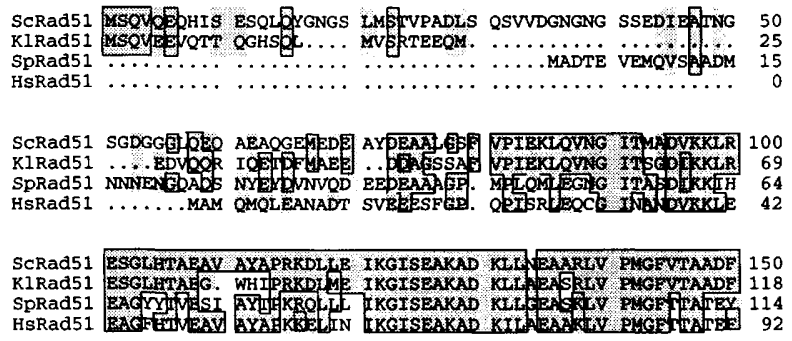

Figure 3. Comparison of $S$. cerevisiae and $K$. lactis Rad51 and the amino termini of Rad51 homologs. (A) The $S$. cerevisiae Rad51 protein (ScRad51) aligned with $K$. lactis Rad51 (KlRad51) using the PileUp program of the Genetics Computer Group (1991). Identity is indicated by shaded boxes; while similarities are shaded without boxes. Amino acid similarity groups (in parenthesis) are $(C),(P),(H),(I, V),(L, M),(K, R),(S, T),(A, G)(F, W$, $\mathrm{Y}),(\mathrm{N}, \mathrm{D}, \mathrm{E}, \mathrm{Q})$. The region of RecA homology is indicated by the line above the sequences. $(B)$ The amino-terminal self-interaction domain of $S$. cerevisiae Rad51 protein (SCRad51) aligned as above with $K$. lactis Rad51 (KlRad51), S. pombe Rad51 (SpRad51), and human Rad51 (HsRad51). Identity and similarities are indicated as described above.

survival over 5 days. KIRAD51 expression resulted in only $10 \%$ survival after 5 days, whereas $R A D 51$ or vector alone showed $100 \%$ survival after only 2 days (Fig. 4B). The dominant-negative effect of KlRAD51 appears to be weaker than that of rad51K-A191, which in the same experiment showed no growth on MMS containing plates even after 5 days (data not shown).

The dominant-negative effect exerted by KlRad51 could result from its interaction with ScRad51. To determine whether KlRad51 and ScRad51 can physically associate, TAKl51 was prepared and cotransformed into GGY1::171 with DB51. Transformants replica-plated onto X-gal plates turned deep blue overnight, and extracts had 414 Miller units of $\beta$-galactosidase activity (Fig. 2A). The interaction of $K I \operatorname{Rad} 51$ with $S c \operatorname{Rad} 51$ is at least as strong as $S c \operatorname{Rad} 51$ self-association.
GAL4-RAD51 fusions are additional dominantnegative alleles of RAD51

We found that Gal4-Rad51 fusions (TA51 and DB51) did not complement the MMS sensitivity of a rad51 $\Delta$ strain (data not shown). Because other mutant proteins, which retain interaction epitopes, dominantly interfered with DSB repair, we examined the phenotype of wild-type cells expressing TA51 and DB51. The wild-type strain DWY127 was transformed with either DB51 or TA51, and DSB repair of the transformants was measured on MMS selection plates. DB51 expression considerably reduced DWY127 survival on MMS plates when compared with a vector only control (Fig. 5A). The effect of DB51 expression was similar to that of rad51K-A191. Likewise, expression of TA51 also strikingly reduced MMS survival of DWY127 (data not shown). Although the expression of TA51 or DB51 also appeared to decrease the growth of GGY1::171, UV sensitivity of cells expressing DB51 was indistinguishable from those with vector only (data not shown). Thus, Gal4-Rad51 fusions must specifically interfere with DSB repair in wild-type yeast.
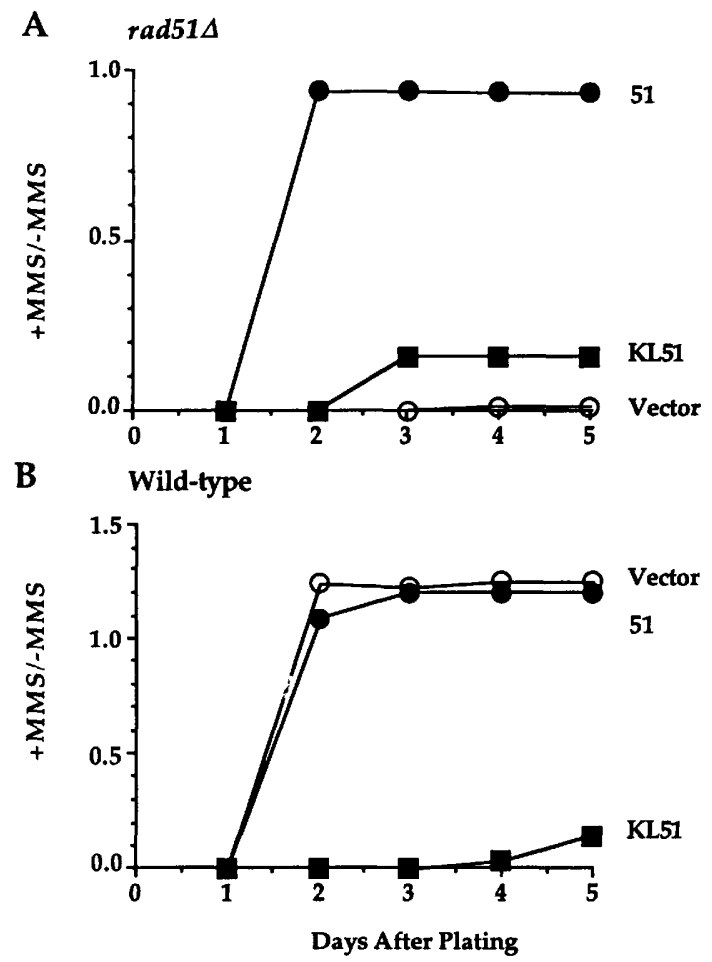

Figure 4. KIRAD51 partially complements a $S$. cerevisiae rad51 $\Delta$ strain and has a dominant-negative effect on MMS repair in a wild-type strain. MMS sensitivity was measured as described in Fig. 1 and Materials and methods. $(A)$ Expression of KlRAD51 partially complements rad51 $\Delta$ cells. MMS sensitivity of DWY129 (rad514) transformed with pDB20 (Vector; O), $A D H: 51(51 ; 0)$, and $p R S K 151(\mathrm{KL} 51 ; \mathbf{\square})$ and assayed on plates lacking uracil with $0.0025 \%$ MMS. (B) Overexpression of KlRAD51 sensitizes wild-type cells to MMS. DWY83 (RAD51) was transformed with pDB20 (Vector; O), $A D H: 51$ (51;O), and $A D H: K 151(\mathrm{KL} 51 ; \mathbf{Q})$ and assayed on plates lacking uracil with $0.0025 \%$ MMS. 
A

wild-type

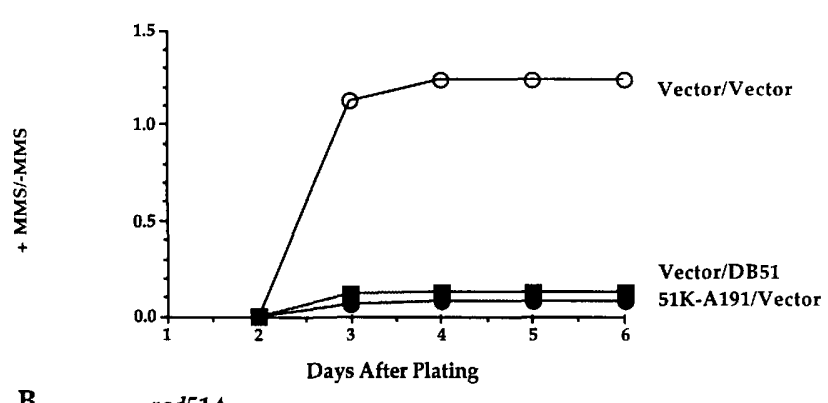

B

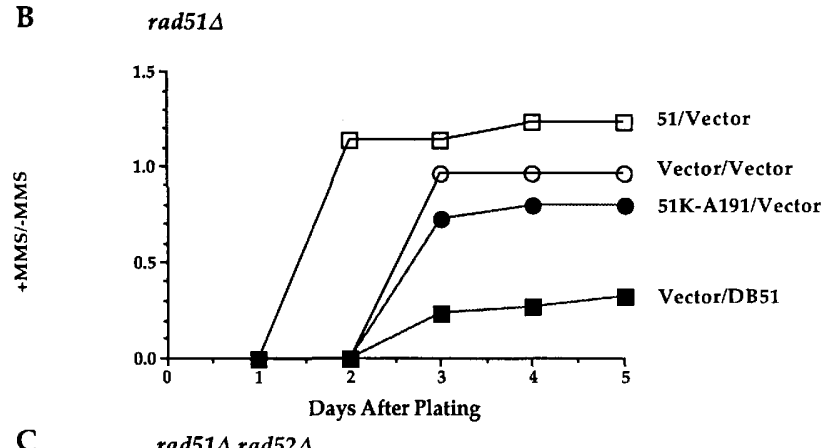

C

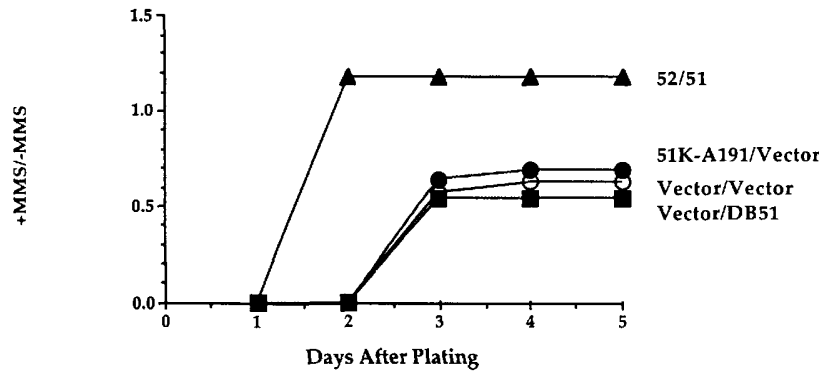

Figure 5. Gal4-Rad51 fusions dominantly interfere with DSB

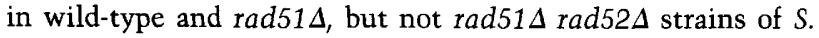
cerevisiae. MMS sensitivity was measured as described in Fig. 1 and Materials and methods. (A) Overexpression of DB51 sensitizes wild-type cells to MMS to approximately the same extent as rad51K-A191. DWY127 (RAD51) was transformed with pDB20 and pMA424 (Vector/Vector; O), pDB20 and DB51 (Vector/DB51; $\mathbf{\square}), A D H 51 K-A 191$ and pMA424 (51K-A191/Vector; O) and assayed on plates lacking uracil and histidine with $0.0025 \%$ MMS. $(B)$ Expression of DB51 increases the MMS sensitivity of a rad51 $\Delta$ strain whereas rad51K-A191 has no effect. DWY129 (rad51 $\Delta$ ) was cotransformed with pDB20 and pGAD10 (Vector/Vector; O), $A D H: 51$ and pGAD10 (51/Vector; $\triangle$ ), $A D H: 51 K-A 191$ and pGAD10 (51K-A191/Vector; O), pDB20 and DB51 (Vector DB51; $\mathbf{0}$ ) and assayed on plates lacking uracil and leucine with $0.0025 \%$ MMS. (C) Expression of DB51 has no effect on the MMS sensitivity of a rad51 $\Delta$ rad52 $\Delta$ strain. DWY144 (rad51 $\Delta$ rad52 4 ) was cotransformed with pDB20 and pMA424 (Vector/Vector; Ol, $A D H: 52$ and pRS51 (52/51; $\Delta$ ), $A D H: 51 K-A 191$ and pMA424 (51K-A191/Vector; O), pDB20 and DB51 (Vector/DB51; $\mathbf{D}$ ) and assayed on plates lacking uracil and histidine with $0.00063 \%$ MMS.

Because Rad51 can both self-associate and interact with Rad52, mutant Rad51 may interfere with DNA repair by nonproductively associating with either or both proteins. To examine this, we assayed the MMS repair

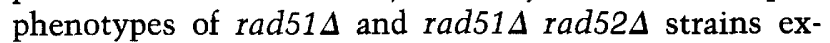
pressing mutant alleles. rad51 $\Delta$ strains are less MMS

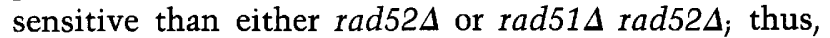
mutant Rad51 that associates nonproductively with Rad52 could increase the MMS sensitivity of a rad51 strain, which has Rad52. A mutant that nonproductively associates with Rad51 would have no effect in this strain. Additionally, if the effect of mutant Rad51 is limited to its interactions with Rad51 and Rad52, there should be no additional effect on MMS sensitivity in a rad51 $\Delta$ rad52 $\Delta$ strain. DNA repair phenotypes of rad51 $\Delta$ and rad51 $\Delta \mathrm{rad} 52 \Delta$ strains were measured on plates with MMS concentrations reduced relative to experiments already described (Materials and methods).

The rad514 strain DWY129 was transformed with $R A D 51$, rad51K-A191, DB51, or vector control and examined for MMS sensitivity. RAD51 fully complemented DWY129. Interestingly, DB51 increased the MMS sensitivity of DWY129, whereas rad51K-A191 exhibited similar MMS sensitivitiy as the vector control (Fig. 5B). TA51 expressed in another rad51A strain (DWY120) also increases MMS sensitivity relative to a vector only control (data not shown). Thus, both TA51 and DB51 can disrupt MMS repair independently of Rad51, whereas rad51K-A191 cannot. We also found that both DB51 and TA51 independently increased the MMS sensitivity of rad52 $\Delta$ strains (where Rad51 is present), whereas rad51K-A191 did not (data not shown). Thus, it appears that TA51 and DB51 can also increase MMS sensitivity in the absence of Rad52.

Next, we tested the MMS repair phenotypes of a

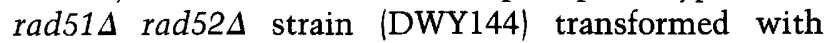

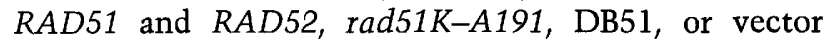
alone. These assays were also performed with reduced MMS concentrations so that hypersensitivity could be scored. Expression of wild-type Rad51 and Rad52 fully complemented the MMS sensitivity of DWY144. In contrast, transformants that expressed rad51K-A191 and DB51 had the same sensitivity as vector alone (Fig. 5C). In total, these results suggest that the level of MMS sensitivity in yeast expressing Gal4-Rad51 fusion proteins depends on the presence of either Rad51 or Rad52. In the absence of both proteins, MMS sensitivity is equal to that of DWY144.

\section{Evidence for other protein components of a Rad51- Rad52 repair complex}

$K$. lactis and $S$. cerevisiae are relatively closely related; thus it is unlikely that the poor complementation of $S$. cerevisiae mutants by KlRAD51 and KlRAD52 (Fig. 4A; and Milne and Weaver 1993) is attributable to functional divergence of their gene products. A more likely explanation is that the protein-protein interaction epitopes have diverged. Because we have observed in vivo physical association of Rad51 and Rad52 from both species (this paper; Milne and Weaver 1993), it is possible that codivergence of these two proteins alone is sufficient to explain the poor complementation. Alternatively, other 
species-specific interactors with Rad51 and Rad52 may be required. KIRAD51 and KIRAD52 were coexpressed in a $S$. cerevisiae rad514 rad524 strain (DWY144), such that full complementation would be expected if only Rad51 and Rad52 act together in DSB repair. For this experiment the genes were expressed from low-copynumber plasmids with their own promoters. Cells cotransformed with KlRAD51 and KIRAD52, KIRAD51 and SCRAD52, ScRAD51 and KIRAD52, and ScRAD51 and $S C R A D 52$ were tested for MMS survival. Although the $S$. cerevisiae genes fully complemented the rad51 $\Delta$ rad52 $\Delta$ strain, coexpression of KlRAD51 and KlRAD52, and the mixtures of RAD51 and RAD52 from S. cerevisiae and $K$. lactis provided only low levels of complementation (Fig. 6). These effects are not likely to arise from the weak activity of $K$. lactis promoters in $S$. cerevisiae because the $K 1 \operatorname{Rad} 51$ and $K 1 \operatorname{Rad} 52$ constructs used in these experiments complemented respective $S$. cerevisiae rad51 $\Delta$ or rad52 $\Delta$ srains nearly as well as KlRad51 and KlRad52 overexpressed from $A D H 2 \mu$ plasmids. Thus, species-specific interaction of Rad51 and Rad52 alone does not provide wild-type level DSB repair in $S$. cerevisiae. This result supports the hypothesis that there are additional proteins that must interact with Rad51 and Rad52 for efficient DSB repair.

\section{Discussion}

Our results with dominant-negative RAD51 alleles are consistent with Rad51 homotypic and heterotypic protein-protein interactions being important for DSB repair. Self-association, as well as $\operatorname{Rad} 51: \operatorname{Rad} 52$ interactions, are mediated by the amino-terminal region of Rad51. These associations appear to be biologically significant because rad51K-A191, K. lactis RAD51, and GAL4-

rad51 1 rad52 4

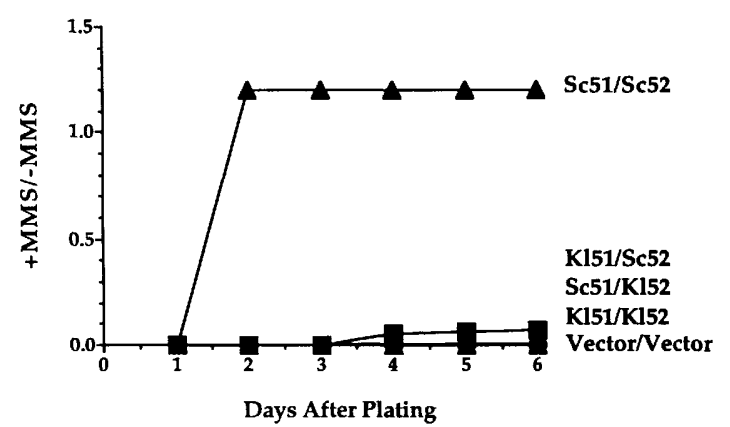

Figure 6. Coexpression of $K$. lactis $\operatorname{Rad} 51$ and $\operatorname{Rad} 52$ homologs in a $S$. cerevisiae rad51 $\Delta$ rad52 $\Delta$ strain does not complement DSB repair. MMS sensitivity was measured as described in Fig.

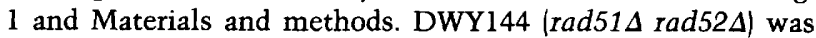
cotransformed with empty vectors (Vector/Vector; O), RAD51 and RAD52 (Sc51/Sc52; $\Delta), K I R A D 51$ and RAD52 (K151/Sc52; च), RAD51 and KIRAD52 (Sc51/K152; $\triangle$ ), KlRAD51 and KIRAD52 (Kl51/K152; ) and assayed on plates lacking uracil and histidine with $0.00375 \%$ MMS.
RAD51 fusions all disrupt DSB repair in wild-type strains. In the case of rad51K-A191, the dominant-negative effect depends solely on the presence of wild-type Rad51, indicating that it blocks DSB repair via its interaction with endogenous Rad51. Two other dominantnegative alleles, GAL4-RAD51 fusions and K1RAD51, have phenotypes indicating that other components of a $\operatorname{Rad} 51 / \operatorname{Rad} 52$ repair complex are likely to be necessary for Rad51 function. The significance of these effects and protein-protein interactions in DSB repair will be discussed.

\section{Rad51 homotypic interaction and RecA homology}

The amino acid similarity between bacterial RecA and Rad51 suggests that these proteins have an evolutionarily conserved role in DNA repair and recombination (Shinohara et al. 1992, 1993). Our results with dominantnegative RAD51 alleles and the identification of an amino-terminal self-interaction epitope for Rad51 are consistent with similar findings for RecA. Carboxy-terminal truncations of RecA dominantly interfere with DNA repair in wild-type bacteria, indicating that the amino-terminal region is likely involved in self-interaction and that the interaction is important for RecA function (Yarranton and Sedgwick 1982; Horii et al. 1992; Tateishi et al. 1992). Amino-terminal deletions of 15 or 28 aminio acids dominantly disrupt wild-type RecA function, whereas a more extensive deletion of 59 amino acids has no effect (Horii et al. 1992). These truncated proteins identify a region of RecA responsible for dominant interference and, therefore, self-interaction. Additionally, RecA missing 33 amino acids at its amino terminus fails to bind ssDNA, apparently because of a reduced ability to self-associate (Ogawa and Ogawa 1986). The RecA crystal structure confirmed that residues between amino acids 15 and 59 lie at the interface between RecA subunits and are likely critical for polymer assembly (Story et al. 1992). From these studies, the ability of mutant RecA to interfere with endogenous RecA activity correlates with RecA self-interaction.

As with bacterial RecA, our mapping data shows that the amino-terminal region of Rad51 is required for homotypic associations. Only truncated Gal4-Rad51 fusions that retain this region can associate with fulllength Rad51 fusions (Fig 2B). Based on the central core of RecA homology, the amino terminus of Rad51 is 120 amino acids longer. Although the region immediately flanking the core homology has limited amino acid similarity to RecA, both proteins have predicted $\alpha$-helical structures in this region (Story et al. 1992, 1993). It is likely that an amino-terminal $\alpha$-helix is involved in the self-association of Rad51 monomers.

KlRad51 and ScRad51 are very similar over their entire lengths; however, the amino-terminal region of KlRad51 is both smaller and relatively divergent from the ScRad51 (Fig. 3). Over the region of Rad51 homotypic interaction (amino acids 1-151), the K. lactis and S. cerevisiae proteins are only $58 \%$ similar. Despite this dif- 
ference, KlRad51 and Rad51 are capable of physically interacting (Fig 2A). The sequence divergence in the region important for protein-protein interactions may explain why KlRad51 only partially complements rad51 $\Delta$ strains and behaves as a dominant-negative allele in wild-type cells (Fig. 4). KIRad51 may not properly associate with $S c$ Rad51 and $S c \operatorname{Rad} 52$, which could result in DSB repair complexes of reduced activity. In contrast to the partially active KlRad51, S. pombe, mouse, and human Rad51 homologs do not complement $S$. cerevisiae rad510 strains at all (Shinohara et al. 1993; Donovan et al., unpubl.). In addition, HuRad51 fails to interact with ScRad51 by two-hybrid analysis and does not exert a dominant-negative phenotype in wild-type yeast (data not shown). These Rad51 homologs are even more divergent from $S$. cerevisiae Rad51 at their amino termini (Fig. 3B). It appears that the increasing divergence of this region in Rad51 homologs limits their ability to interact with endogenous Rad51 and complement rad51 defects.

Only truncated Gal4-Rad51 fusions containing the amino-terminal region associate with full-length $\mathrm{Rad} 51$ (Fig. 2B). We also tested combinations of the truncated fusions to determine which regions of both $\operatorname{Rad} 51$ partners were needed for association. Only Rad51 fusion proteins containing the amino-terminal region can interact with other truncated fusions. For example, 51A will interact with 51, 51A, 51C, 51D, 51E, and 51G but not with $51 \mathrm{~B}$ or $51 \mathrm{~F}$ (data not shown). This suggests that Rad51 monomers interact in a head-to-head manner. However, the packing of RecA seen in the crystal structure is a head-to-tail association, where $\beta$-strand 0 and $\alpha$-helix A of one molecule contact $\beta$-strand 3 and $\alpha$-helix $E$ of an adjacent molecule in an antiparallel fashion (Story et al. 1992). Depending on how the sequences are aligned, the corresponding region of $\operatorname{Rad} 51$ would be somewhere between amino acids 140 and 170. This region, however, is absent in several of the Gal4-Rad51 fusion proteins that interact $(51 \mathrm{~A}, 51 \mathrm{C}, 51 \mathrm{G})$, and present in the truncated fusions $51 \mathrm{~B}$ and $51 \mathrm{~F}$ that do not interact. Therefore, Rad51 and RecA complexes may be fundamentally different in their packing structure, despite the striking physical similarities observed between their nucleoprotein filaments (Ogawa et al. 1993). Alternatively, the association of Rad51 with DNA may order the packing in a manner that we cannot observe by fusion protein associations. The two-hybrid assay presumably only requires dimerization for detection. $\operatorname{Rad} 51: \operatorname{Rad} 51$ dimers may not require all of the interactions necessary for filament formation on DNA. It is also formally possible that the two-hybrid assay is not measuring direct self-association but that another factor in the yeast nucleus facilitates the interaction of Gal4Rad51 fusion proteins. Endogenous Rad51, which is present in the GGY1::171, is a likely candidate for this factor. We tested the possibility that endogenous Rad51 was influencing the two-hybrid results. Rad51 homotypic association was unchanged in a rad51 $\Delta$ derivative of GGY1::171 (data not shown). Thus, the self-interaction observed is neither dependent on nor influenced by endogenous Rad51.
rad51K-A191 exerts its effect through homotypic associations

The simplest explanation for the phenotypes produced by dominant-negative $R A D 51$ alleles is that mutant Rad51 nonproductively associates with endogenous proteins involved in DSB repair. Our data indicate that the interaction of endogenous Rad51 with mutant Rad51KA191 and KlRad51 drives dominant-negative DNA repair phenotypes. The values for $\beta$-galactosidase activity by two-hybrid analysis demonstrate strong and/or stable Rad51:Rad51K-A191 and Rad51:KIRad51 interactions. These alleles both impair DSB repair when expressed in wild-type strains (Figs. 1 and 4). Also, the rad51K-A191 allele depends on endogenous Rad51 to exert a dominant negative effect because DSB repair is not reduced by the addition of Rad51K-A191 to a rad51 $\Delta$ strain (Fig. 5).

Two mechanisms could describe the nonproductive association of mutant Rad51 with DSB repair complexes. First, the overexpression of altered Rad51 may disrupt the formation of repair complexes by occupying all Rad51 protein-protein interaction sites. Instead of forming extended Rad51 complexes, these associations could primarily create inactive homo- or heterodimers or lower-order multimers of Rad51 that are too small to be functionally significant. Alternatively, mutant and wildtype Rad51 may associate to form extended, but mixed complexes that have defective DSB repair activity. Similar mechanisms have been proposed for the dominant interference of RecA function by truncated $r e c A$ alleles (Yarranton and Sedgwick 1982; Horii et al. 1992).

Rad51K-A191 has no additional DSB repair phenotype in a rad52 $\Delta$ strain even though this strain has wild-type Rad51 (data not shown). Considering that rad52 $\Delta$ strains are more MMS sensitive than rad51 $\Delta$ strains, it is possible that interference of Rad51 function cannot be detected in a rad52 $\Delta$ strain. However, DB51 and TA51 did show dominant-negative phenotypes in both rad51 $\Delta$ and

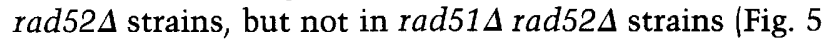
and data not shown). Thus, functionally significant Rad51:Rad51 associations may also be dependent on Rad52 activity to be manifest. Rad51K-A191 may form inactive filaments with endogenous $\operatorname{Rad} 51$, whereas DB51 and TA51 may not form filaments at all, but force nonproductive interactions with Rad52.

\section{Rad51 heterotypic interactions}

We demonstrated previously that $\operatorname{Rad} 51$ and $\operatorname{Rad} 52$ interact in vivo and that this interaction is important for DSB repair in S. cerevisiae (Milne and Weaver 1993). Using truncated Gal4-Rad51 fusions, we found that Rad52:Rad51 interaction also maps to the amino-terminal region of Rad51 (Fig. 2B). Rad51 self-association and the weaker Rad51:Rad52 interaction are separable for two of the truncated Gal4-Rad51 fusions (51D and 51G) but are otherwise identical. Because of the close proximity of $\operatorname{Rad} 51: \operatorname{Rad} 51$ and $\operatorname{Rad} 51: \operatorname{Rad} 52$ interactions, Rad51 self-association may be influenced by Rad52. We tested the Rad52 dependence of Rad51 self-association in 
a rad52 $\Delta$ derivative of GGY1::171. TA51 and DB51 coexpression continued to produce a strong signal for $1 a c Z$ expression, indicating that Rad51 self-association is independent of Rad52 (data not shown).

Our data indicate that $\operatorname{Rad} 51: \operatorname{Rad} 51$ and $\operatorname{Rad} 51: \operatorname{Rad} 52$ associations are not sufficient for DSB repair. The coexpression of $K l \operatorname{Rad} 51$ and $K l \operatorname{Rad} 52$ in a $S$. cerevisiae rad51 $\Delta$ rad52 $\Delta$ strain does not significantly restore DSB repair (Fig. 6). KIRad51 and $K I$ Rad52 are active proteins in $S$. cerevisiae, however, because each is able to partially complement a respective null mutant (Fig. 4; Milne and Weaver 1993). It is unlikely that their lack of complementation is attributable to the weak activity of $K$. lactis promoters in S. cerevisiae. Both KIRAD51 and KlRAD52 showed only slightly better complementation in their respective single deletion strains when expressed from the powerful $S$. cerevisiae $A D H$ promoter. Moreover, Western blots using anti-Rad52 antisera show that levels of KIRad52 produced in a $S$. cerevisiae rad52 strain when it was expressed from the $K$. lactis promoter were comparable to that of $S c \operatorname{Rad} 52$ in wild type (data not shown). Our interpretation of these results is that additional species-specific components are required to modulate Rad51/Rad52 functions. At least some of these additional components may be mediated by protein-protein associations. Other $S$. cerevisiae RecA homologs could be candidates for additional Rad51 interacting proteins. Rad55, Rad57, and Dmcl are also structurally related to RecA and function in DNA repair and recombination (Kans and Mortimer 1991; Bishop et al. 1992; Lovett 1994). We have been unable to detect the interaction of Rad51 with either Rad57 or Dmc1 by the twohybrid methodology, indicating that these interactions are either weak and not detectable by our fusion proteins or that these proteins do not physically interact in yeast (data not shown).

Dominant-negative alleles of Rad51 have demonstrated the importance of protein-protein interactions in DSB repair in yeast. Dominant-negative alleles may also be useful for determining the role Rad51 homologs have in DSB repair and recombination in mammalian cells. We are examining the effect of overexpressing a human rad51 allele with a missense mutation in its ATP-binding site in mammalian cells.

\section{Materials and methods}

Strains, media, and genetic methods

All yeast strains used in this study are shown in Table 1. DWY120 is a 5-fluoro-orotic acid (5-FOA) selected clone of NKY1826. DWY144, a rad514 rad524 strain, is a haploid segregant of the diploid strain DWY140 constructed by mating DWY97 (Milne and Weaver 1993) with DWY129. Yeast strains were grown at $30^{\circ} \mathrm{C}$ in appropriate media as described previously (Sherman et al. 1983). Liquid media used were YPD ( $1 \%$ yeast extract, $2 \%$ Bacto-peptone, $2 \%$ dextrose) for rapid growth, SC media lacking amino acids for plasmid selection $10.67 \%$ yeast nitrogen base, $2 \%$ dextrose, without the appropriate amino acids). Solid media was prepared by adding Bacto-agar to $2.0 \%$. Transformations were performed as described previously [Gietz et al. 1992), with the addition of dimethylsalfoxide
(DMSO) up to $10 \%$ before heat shock as described by (Hill et al. 1992).

\section{Bacterial plasmids}

Four RAD51 subclones were prepared that served as parent molecules for all further RAD51 constructs. p51SP was created by ligation of the $1.5 \mathrm{~kb}$ genomic StuI-Pst I fragment, which contains the entire RAD51 open reading frame into BamHI (filledin)-PstI cut $\mathrm{pSKII}^{+}$. p51B, used to build GAL4 fusion constructs, was made in multiple steps. A polymerase chain reaction (PCR) fragment amplified from a genomic clone with the degenerate primers $51 \mathrm{~N}$ (5'-GCGGATCCA(A/G)GA(A/G)C$\left.\mathrm{A}(\mathrm{A} / \mathrm{G}) \mathrm{CA}(\mathrm{C} / \mathrm{T}) \mathrm{AT}-3^{\prime}\right)$ and 51C (5'GCGGATCC $(\mathrm{C} / \mathrm{T}) \mathrm{TC}(\mathrm{A} /$ G)TC(C/T)TC(T/C)TC-3'), was digested with BamHI and ligated to BamHI-cut pSKII ${ }^{+}$. The 1-kb BamHI-BstXI fragment

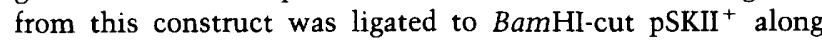
with a 500-bp BstXI-EcoRV-BamHI-linkered fragment from p51SP. p51B, therefore, contains sequences that encode all but the four amino-terminal amino acids of the Rad51 protein along with $300 \mathrm{bp}$ of downstream noncoding sequence from p51SP. p51BK-A191 was constructed in two steps. A PCR fragment was amplified from p51SP using the primers $51 \mathrm{~K}-\mathrm{A}\left(5^{\prime}\right.$-GGTGAATTCAGGACAGGTGCGTCCCAGCTATG-3') and 51C (described above), digested with EcoRI and $B a m H I$, and ligated to EcoRI-BamHI-cut pSKII ${ }^{+}$. An EcoRI-PvuII fragment from this intermediate was ligated to the EcoRI-HincII-digested backbone of $\mathrm{p} 51 \mathrm{~B}$ to give $\mathrm{p} 51 \mathrm{BK}-\mathrm{A} 191$. This construct contains two nucleotide changes in the coding sequences that result in a substitution of lysine to alanine at position 191. p51SPK-A191 was made by ligating an EcoRI-XhoI fragment from p51BK-A to the EcoRI-Xhol-digested backbone of p51SP. This construct contains the K-A19l mutation in the context of the entire RAD51 open reading frame.

\section{Two-hybrid fusion protein plasmids}

Plasmids for the GAL4 two-hybrid fusion assay were made by subcloning various fragments from $\mathrm{p} 51 \mathrm{~B}$ and $\mathrm{p} 51 \mathrm{SP}$ into pMA424 (containing amino acids 1-147 of the DNA-binding domain of GAL4) and pGAD2F and pGAD10 (containing amino acids 768-881 from the trans-activation domain of GAL4; Chien et al. 1991). pMA51 (DB51) and pGAD51 (TA51) were made by ligating the entire $B a m H I$ insert from p5lB into the BamHI site of pMA424 and pGAD2F. pMA51A and pGAD51A were built by ligating a 560-bp BamHI-EcoRI-BamHI linkered fragment from p51B into BamHI-cut pMA424 and pGAD2F. pMA51B and pGAD51B were made by ligating a $0.9-\mathrm{kb}$ EcoRI fragment from p51SP, containing the carboxy-terminal half of $R A D 51$, into the EcoRI site of pMA424 and pGAD10. pMA51C and pGAD51C were made by filling in the 1250-bp Bsu36IEcoRV fragment from p51SP and ligating to BamHI-digested and filled-in pMA424 and pGAD2F. pGAD51D was constructed by filling in the 330-bp Bsu36I-EcoRI fragment from p51B and ligating it to BamHI-cut and -blunted pGAD2F. pMA51E was made from a subclone of $\mathrm{p} 51 \mathrm{~B}$ in which the 120-bp NdeI-NdeI fragment was cut out, and the NdeI ends filled-in and religated. This construct thus has an in-frame deletion of 39 amino acids. pMA51D contains the BamHI insert from this intermediate ligated to BamHI-cut pMA424. pMA51F was made by filling in the 1-kb NdeI-BamHI fragment from $\mathrm{p} 51 \mathrm{~B}$ and ligating it to pMA424 that had been cut with EcoRI and filled in. pMA51G contains the 325-bp NdeI-NdeI fragment from p51SP filled in and ligated to EcoRI-cut and filled-in pGAD10. The fusion protein produced by this construct contains the four amino-terminal amino acids of Rad51 that all others lack. pMA51H was 
made in several steps. A DraI complete-NdeI partial fragment of p51B was filled in and ligated to blunted Bsu36I-EcoRV-cut backbone of p51SP. This intermediate construct contains an in-frame deletion of the 35 amino acids encoded between the Bsu36I and NdeI sites. The coding sequences of the intermediate were amplified by PCR using the primers $51 \mathrm{~N}$ and $51 \mathrm{C}$ described above, and the BamHI-digested PCR product was ligated to BamHI-cut pMA424 to give pMA51H. pMA51K-A191 and pGAD51K-A191 were made by inserting the BamHI insert from p51BK-A191 into BamHI site of pMA424 and pGAD2F. All GAL4-RAD51 fusion constructs were sequenced to ensure that the fusions were correct and in-frame.

pMA52 (DB-52) and pGAD52 (TA-52) have been described elsewhere (Milne and Weaver 1993). pMAKL51 was built by PCR amplifying the coding sequences of a genomic subclone of the $K$. lactis RAD51 gene (described below) using the primers $k 151 \mathrm{~N}\left(5^{\prime}\right.$-GCGGATCCAGGTGGAAGAAGTTCAG-3') and k151C ( $5^{\prime}$-GCGGATCCAGATCAATCATCGTCTTC-3'). This PCR product was digested with $\mathrm{BamHI}$ and ligated to $\mathrm{BamHI}-$ digested pMA424.

\section{Yeast expression plasmids}

S. cerevisiae and $K$. lactis RAD51 alleles were overexpressed in $S$. cerevisiae with the $E$. coli/S. cerevisiae shuttle vector $\mathrm{pDB} 20$ (Becker et al. 1986). This expression vector contains $U R A 3$, a $2 \mu$ origin, and the $A D H I$ promoter and terminator flanking HindIII, NotI, and BstXI sites. pDBRAD51 (51) was constructed by filling in a $X b a I-E c o R V$ fragment from $\mathrm{p} 51 \mathrm{SP}$ and ligating this to the filled-in HindIII site of pDB20. pDB51K-A191 (51K-A191) was built by filling-in an $\mathrm{XbaI}$ fragment from p51SPK-A191 and ligating to HindIII-cut and filled-in pDB20. pDBKL51 was made by ligating a filled-in $1.8-\mathrm{kb}$ genomic AseI fragment from $\mathrm{p} K L 51 \mathrm{HX}$ (described below) to the filled-in HindIII site of pDB20. Single-copy expression of $S$. cerevisiae RAD51 and $K$. lactis RAD51 and RAD52 was achieved with the E. coli/S. cerevisiae shuttle vectors pRS313 and pRS316 (Sikorski and Hieter 1989). These vectors contain HIS3 and URA3 sequences, respectively, along with CEN6 and ARSH4 sequences. pRSSC51 was constructed by ligating the $B a m H I$ genomic fragment containing the $S$. cerevisiae RAD51 gene to BamHI-cut pRS313. pRSKI51 was made by ligating the HindIII-XbaI genomic fragment containing the $K$. lactis RAD51 gene to EcoRV-cut and -blunted pRS313. pRSK152 was constructed by ligating the XbaI genomic fragment containing the $K$. lactis RAD52 gene to SpeIcut pRS316. Single-copy expression of $S$. cerevisiae $R A D 52$ was achieved with YCp52. This construct has a Sall fragment containing $S$. cerevisiae RAD52 filled in and ligated to EcoRI$B a m H I-c u t$ and filled-in backbone from YCpGal:RI (Barnes and Rine 1985| and has the URA3 gene and ARS1 and CEN4 sequences.

\section{Cloning and sequencing $\mathrm{K}$. lactis RAD51}

Genomic DNA from $K$. lactis (ATCC 8585) was digested with $X b a \mathrm{I}$ and size fractionated on a $1 \%$ agarose gel, and DNA fragments were glass bead purified and ligated to $\mathrm{XbaI}$-cut $\mathrm{pKSII}^{+}$. Approximately $4100 \mathrm{E}$. coli DH5 $\alpha$ colonies were screened by low-stringency hybridization $130 \%$ formamide, $1 \times$ Denhardt's solution, $1 \%$ SDS, $0.75 \mathrm{M} \mathrm{NaCl}, 50 \mathrm{mM} \mathrm{NaH}_{2} \mathrm{PO}_{4}, 5 \mathrm{~mm}$ EDTA, and $100 \mathrm{mg} / \mathrm{ml}$ of sheared salmon sperm DNA at $42^{\circ} \mathrm{C}$ ) with a $3.7-\mathrm{kb}$ BamHI fragment that contains the entire $S$. cerevisiae $R A D 51$ gene. Three 9-kb clones that have a common restriction map were identified. The $K$. lactis RAD51 open reading frame was sequenced using Sequenase 2.0 (U.S. Biochemical) by standard procedures.

\section{GAL4 two-hybrid interaction assay}

The GAL4-RAD51 fusion constructs described above were cotransformed into GGY1::171. Transformants were incubated $2-10$ days at $30^{\circ} \mathrm{C}$ until colonies were fairly large. $\beta$-Galactosidase production was determined initially by replica-plating colonies onto plates containing X-gal (GIBCO) and then quantitated by a liquid assay (Chien et al. 1991). This was modified so that the assay was performed on cells scraped from selective plates because of differences in growth that appear to result from the expression of Rad51 fusion proteins.

\section{MMS cell survival assay}

The MMS phenotype of strains expressing various RAD51 alleles was measured essentially as described (Milne and Weaver 1993). Yeast colonies were picked in triplicate into $\mathrm{H}_{2} \mathrm{O}$, diluted $1: 10$, and serially diluted (six fivefold serial dilutions). Aliquots $(10 \mu l)$ of each dilution were spotted in duplicate on selective plates with or without the appropriate concentration of MMS. Plates were incubated at $30^{\circ} \mathrm{C}$, and colonies were counted daily for 5 days. The relative resistance of strains is shown as the daily ratio of the number of colonies on MMS plates relative to the total number of colonies appearing on the non-MMS control plates. This number represents an average of the three individual transformants picked for each strain. The concentration of MMS used to measure the dominant negative effect of mutant alleles in the wild-type strains DWY83 and DWY127 was $0.0025 \%$. The concentration of MMS used in the rad51 $\Delta$ strains DWY120 and DWY129 to assess complementation of various alleles was $0.005 \%$. The dominant effect of various alleles expressed in rad51 $\Delta$ strain DWY120 was measured on plates that were $0.0025 \%$ MMS. The concentration of MMS used to measure dominant-negative effects of alleles expressed in rad51 rad52 2 strain DWY144 was $0.00063 \%$.

\section{Acknowledgments}

We thank T. Orr-Weaver for critical reading of the manuscript and members of the Weaver laboratory for many helpful discussions. S. cerevisiae strains were kindly provided by N. Kleckner, R. Kolodner and R. Rothstein. We are grateful to S. Fields for strains and plasmids for the GAL4 two-hybrid fusion analysis. This work was supported by a Barr Program grant to D.T.W.

The publication costs of this article were defrayed in part by payment of page charges. This article must therefore be hereby marked "advertisement" in accordance with 18 USC section 1734 solely to indicate this fact.

\section{References}

Aboussekhra, A., R. Chanet, A. Adjiri, and F. Fabre. 1992. Semidominant suppressors of Srs2 helicase mutations of Saccharomyces cerevisiae map in the RAD51 gene, whose sequence predicts a protein with similarities to procaryotic RecA protein. Mol. Cell. Biol., 12: 3224-3234.

Bardwell, A., L. Bardwell, D. Johnson, and E. Friedberg. 1993. Yeast DNA recombination and repair proteins Rad1 and Rad10 constitute a complex in vivo mediated by localized hydrophobic domains. Mol. Microbiol. 8: 1177-1188.

Barnes, G. and J. Rine. 1985. Regulated expression of endonuclease EcoRI in Saccharomyces cerevisiae: Nuclear entry and biological consequences. Proc. Natl. Acad. Sci. 82: 1354-1358.

Basile, G., M. Aker, and R. Mortimer 1992. Nucleotide sequence and transcriptional regulation of the yeast recombinational 
repair gene RAD51. Mol. Cell. Biol. 12: 3235-3246.

Becker, D.M., J. Fikes, and L. Guarente. 1986. A cDNA encoding a human CCAAT-binding protein by functional complementation in yeast. Proc. Natl. Acad. Sci. 88: 1968-1972.

Bell, S. and B. Stillman. 1992. ATP-dependent recognition of eukaryotic origins of DNA replication by a multiprotein complex. Nature 357: 128-134.

Bezzubova, O., A. Shinohara, R.G. Mueller, H. Ogawa, and J.M. Buerstedde. 1993. A chicken RAD51 homologue is expressed at high levels in lymphoid and reproductive organs. Nucleic Acids Res. 7: 1577-1580.

Bishop, D., D. Park, L. Xu, and N. Kleckner. 1992. DMC1: A meiosis-specific yeast homolog of $r e c A$ required for meiotic recombination, synaptonemal complex formation, and cell cycle progression. Cell 69: 439-456.

Chien, C., P.L. Bartel, R. Sternglanz, and S. Fields. 1991. The two-hybrid system: A method to identify and clone genes for proteins that interact with a protein of interest. Proc. Natl. Acad. Sci. 88: 9578-9582.

Friedberg, E. 1991. Yeast genes involved in DNA repair processes: New looks at old faces. Mol. Microbiol. 5: 23032310.

Game, J. 1983. Radiation-sensitive mutants and repair in yeast. In Yeast genetics; fundamental and applied aspects (ed. J. Spencer, D. Spencer and A. Smith), pp. 105-137. SpringerVerlag, New York.

Gietz, D., A.S. Jean, R.A. Woods, and R.H. Schiestl. 1992. Improved method for high efficiency transformation of intact yeast cells. Nucleic Acids Res. 20: 1425.

Hill, J., K.A. Ian, G. Donald, and D.E. Griffiths. 1992. DMSOenhanced whole cell yeast transformation. Nucleic Acids Res. 19: 5791.

Horii, T., N. Ozawa, T. Ogawa, and H. Ogawa. 1992. Inhibitory effects of $\mathrm{N}$ - and C-terminal truncated Escherichia coli recA gene products on functions of the wild-type recA gene. $J$. Mol. Biol. 223: 105-114.

Jang, Y.K., Y.H. Jin, E.M. Kim, F. Fabre, S.H. Hong, and S.D. Park. 1994. Cloning and sequence analysis of RHP51+, a Schizosaccharomyces pombe homolog of the Saccharomyces cerevisiae RAD51 gene. Gene 142: 207-211.

Kans, J. and R. Mortimer. 1991. Nucleotide sequence of the RAD57 gene of Saccharomyces cerevisiae. Gene 105: 139140.

Kornberg, A. and T. Baker. 1992. DNA replication, 2nd ed. Freeman, New York.

Kowalczykowski, S.C. 1991. Biochemical and Biological function of Escherichia coli RecA protein: Behavior of mutant RecA proteins. Biochimie 73: 289-304.

Lamond, A. 1993. The spliceosome. Bioessays 15: 595-603.

Li, J. and B. Alberts. 1992. Eukaryotic initiation rites. Nature 357: 114-115.

Lovett, S.T. 1994. Sequence of the RAD55 gene of Saccharomy. ces cerevisiae: Similarity of RAD55 to prokaryotic RecA and other RecA-like proteins. Gene 142: 103-106.

McHenry, C. 1991. DNA polymerase III holoenzyme. J. Biol. Chem. 266: 19127-19130.

Milne, G.T. and D.T. Weaver. 1993. Dominant-negative alleles of RAD52 reveal a DNA repair/recombination complex including Rad51 and Rad52. Genes \& Dev. 7: 1755-1765.

Modrich, P. 1991. Mechanisms and biological effects of mismatch repair. Annu. Rev. Genet. 25: 229-253.

Morita, T., Y. Yoshimura, A. Yamamoto, K. Murata, M. Mori, H. Yamamoto, and A. Matsuhiro. 1993. A mouse homolog of the Escherichia coli recA and Saccharomyces cerevisiae RAD51 genes. Proc. Natl. Acad. Sci. 90: 6577-6580.

Munn, M. and B. Alberts. 1991. The T4 polymerase accessory proteins form an ATP-dependent complex on a primer-template junction. I. Biol. Chem. 266: 20024-20033.

Muris, D.F., K. Vreeken, A.M. Carr, B.C. Broughton, A.R. Lehmann, P.H. Lohman, and A. Pastink. 1993. Cloning the RAD51 homologue of Schizosaccharomyces pombe. $\mathrm{Nu}$ cleic Acids Res. 21: 4586-4591.

Ogawa, H. and T. Ogawa. 1986. General recombination: Functions and structure of RecA protein. Adv. Biophys. 21: 135148.

Ogawa, T., X. Yu, A. Shinohara, and E. Egelman. 1993. Similarity of the yeast RAD51 filament to the bacterial RecA filament. Science 259: 1896-1899.

Petes, T., R. Malone, and L. Symington. 1991. Recombination in yeast. In The molecular and cellular biology of the yeast Saccharomyces (ed. J. Broach, J. Pringle, and E. Jones), pp. 407-521. Cold Spring Harbor Laboratory Press, Cold Spring Harbor, New York.

Radding, C.M. 1991. Helical interactions in homologous pairing and strand exchange driven by RecA protein. $l$. Biol. Chem. 266: 5355-5358.

Roca, A.I., and M.M. Cox. 1990. The RecA protein: Structure and function. CRC Crit. Rev. 25: 415-456.

Sancar, A. and G. Sancar. 1988. DNA repair enzymes. Annu. Rev. Biochem. 57: 29-67.

Sherman, F., G. Fink, and J. Hicks. 1983. Methods in yeast genetics. Cold Spring Harbor Laboratory, Cold Spring Harbor, New York.

Shinohara, A., H. Ogawa, and T. Ogawa. 1992. Rad51 protein involved in repair and recombination in $S$. cerevisiae is a RecA-like protein. Cell 69: 457-470.

Shinohara, A., H. Ogawa, Y. Matsuda, N. Ushio, K. Ikeo, and T. Ogawa. 1993. Cloning of human, mouse and fission yeast recombination genes homologous to $R A D 51$ and recA. $\mathrm{Na}$ ture Genetics 4: 239-243.

Sikorski, R.S. and P. Hieter. 1989. A system of shuttle vectors and yeast host strains designed for efficient manipulation of DNA in Saccharomyces cerevisiae. Genetics 122: 19-27.

Stasiak, A., E.H. Egelman, and P. Howard-Flanders. 1988. Structure of helical RecA-DNA complexes. III. The structural polarity of RecA filaments and functional polarity in the RecAmediated strand exchange reaction. $J$. Mol. Biol. 202: 659662.

Story, R., I. Weber, and T. Steitz 1992. The structure of the $E$. coli RecA protein monomer and polymer. Nature 355: 318 325.

Story, R.M., D.K. Bishop, N. Kleckner, and T.A. Steitz. 1993. Structural relationship of bacterial RecA proteins to recombination proteins from bacteriophage $\mathrm{T} 4$ and yeast. Science 259: 1892-1896.

Tateishi, S., T. Horii, T. Ogawa, and H. Ogawa. 1992. C-terminal truncated Escherichia coli RecA protein RecA5327 has enhanced binding affinities to single- and double-stranded DNAs. I. Mol. Biol. 223: 115-129.

Yarranton, G.T., and S.G. Sedgwick. 1982. Cloned truncated recA genes in E. coli. Mol. Gen. Gent. 185: 99-104.

Yoshimura, Y., T. Morita, A. Yamamoto, and A. Matsuhiro. 1993. Cloning and sequence of the human recA-like gene cDNA. Nucleic Acids Res. 21: 1665.

Young, M., M. Reddy, and P. von Hippel. 1992. Structure and function of the T4 DNA polymerase holoenzyme. Biochemistry 31: 8675-8690. 


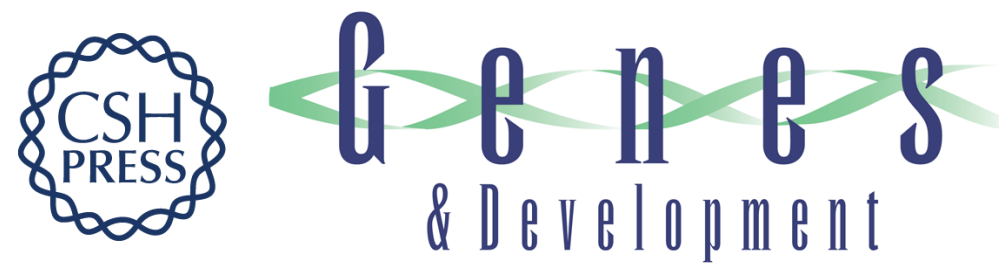

\section{Homotypic and heterotypic protein associations control Rad51 function in double-strand break repair.}

J W Donovan, G T Milne and D T Weaver

Genes Dev. 1994, 8:

Access the most recent version at doi:10.1101/gad.8.21.2552

References This article cites 40 articles, 12 of which can be accessed free at:

http://genesdev.cshlp.org/content/8/21/2552.full.html\#ref-list-1

License

Email Alerting

Service

Receive free email alerts when new articles cite this article - sign up in the box at the top right corner of the article or click here.

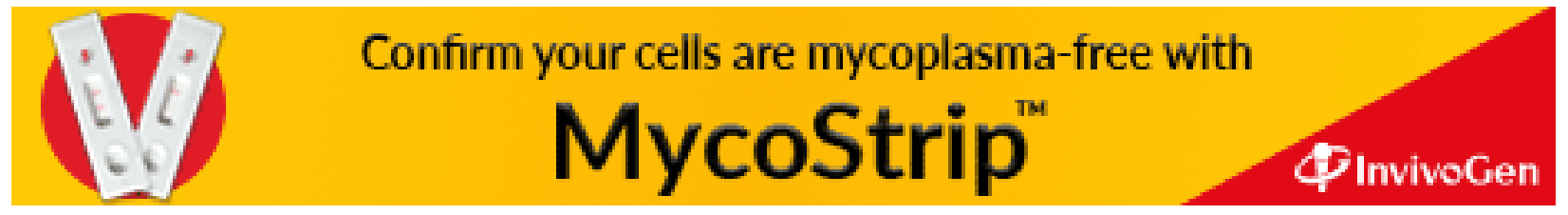

